\begin{tabular}{|c|l|}
\hline Title & $\begin{array}{l}\text { Effects of concentration and temperature on the formation process of decanethiol self-assembled monolayer on Au(111) } \\
\text { followed by electrochemical reductive desorption }\end{array}$ \\
\hline Author(s) & Han, Y Ying; Uosaki, Kohei \\
\hline Citation & $\begin{array}{l}\text { Electrochimica A cta, 53(21), 6196-6201 } \\
\text { https://doi.org/10.1016/.electacta.2008.04.016 }\end{array}$ \\
\hline Issue Date & 2008-09-01 \\
\hline Doc URL & http://hdl.handle.net/2115/39958 \\
\hline Type & article (author version) \\
\hline File Information & EA 53-21_6196.pdf \\
\hline
\end{tabular}

Instructions for use 


\title{
Effects of Concentration and Temperature on the Formation Process of Decanethiol self-assembled monolayer on Au(111) Followed by Electrochemical Reductive Desorption
}

\author{
Ying Han and Kohei Uosaki*
}

Physical Chemistry Laboratory, Division of Chemistry, Graduate School of Science, Hokkaido University, Sapporo 060-0810, Japan

\begin{abstract}
The formation process of self-assembled monolayer (SAM) of decanethiol (C10SH) on $\mathrm{Au}(111)$ single crystal electrode has been investigated for wide range of $\mathrm{C} 10 \mathrm{SH}$ concentration $(0.1 \mu \mathrm{M} \sim 500 \mu \mathrm{M})$ and temperature $(253 \mathrm{~K} \sim 298 \mathrm{~K})$. The amount and quality of C10SH SAM were determined from area and position, respectively, of reductive desorption peak of the SAM modified $\mathrm{Au}(111)$ electrode measured in $0.5 \mathrm{M}$ $\mathrm{KOH}$ aqueous solution. The kinetic analysis indicates that SAM formation proceeded by two steps, diffusion limited physisorption followed by chemisorption.
\end{abstract}

Keywords: self-assembled monolayer, reductive desorption, formation kinetic, thiol, gold single crystal.

* To whom correspondence should be addressed. Phone: +81-11-706-3128. Fax:

+81-11-706-3440. E-mail: uosaki@pcl.sci.hokudai.ac.jp. 


\section{Introduction}

Self-assembled monolayers (SAMs) have been studied by many research groups working in wide variety of fields, both fundamental and applied, such as surface science, biosensors, and biomolecular and molecular electronic devices. Thiol SAMs on gold are the most studied system among the numerous studies on SAMs on oxide, metals, and semiconductors [1-8].

For the establishment of the technique to prepare a stable SAM, it is essential to understand the formation process SAMs. Formation processes of thiol SAMs have been studied using various techniques such as electrochemistry [7], infrared (IR) spectroscopy [9-11], quartz crystal microbalance (QCM) [12-15], scanning tunneling microscopy (STM) [16-18], atomic force microscopy (AFM) [19, 20], second harmonic generation spectroscopy (SHG) [21-23], sum frequency generation (SFG) [24, 25], ellipsometry [26, 27], contact angle measurement [26, 27], and surface plasmon resonance (SPR) [28-30]. Several previous reports suggested that the SAM formation is proceeded first by rapid adsorption followed by slow reorganization $[12-14,26]$. The first kinetic study on alkanethiol SAM formation process on gold was by Bain et al. using ellipsometry and contact angle measurement [26]. They reported that adsorption process was characterized by two distinct steps and that in the first step the coverage reached to $0.8-0.9$ within few minutes but following slower step lasted several hours. We also showed that the formation process of ferrocenylundencanethiol on gold was consisted of two steps and the rate of second step was slower than first step by 2 orders of magnitude using QCM [12]. In contrast, Buck et al. showed that SAM formation process followed the simple Langmuir adsorption kinetics using SHG [21].

Later many papers on more quantitative study for thiol SAM formation have been published [13-15, 20-25, 27-34] but there are some conflicts on kinetic model for the 
thiol adsorption process. Karpovich and Blanchard have shown that the SAM formation can be described by the Langmuir adsorption/desorption kinetics [13]. Campbell and Mutharasan also found that the adsorption can be described by the reversible first-order Langmuir kinetics even at the lowest thiol concentration investigated, i.e., $1 \mathrm{nM}$ [32]. In contrast to these result, Subramanian and Lakshminarayanan suggested that adsorption process is controlled by the first-order and the diffusion-limited Langmuir kinetics at high and low $(<5 \mu \mathrm{M})$ concentration, respectively [31]. Recently Damos et al. suggested the two-step SAM formation process, where thiol molecules first adsorbed on solid surface and then rearranged to become a packed monolayer [30].

Since Widrig et al. reported that an alkanethiol SAM is desorbed from gold surface by a one-electron electrochemical reduction process in an alkaline aqueous solution [35], many electrochemical studies on this reaction have been carried out [34-41]. From the area, shape, and position of the cathodic peak corresponding to reductive desorption of the SAM, one can obtain much information on the SAM such as the amount adsorbed thiol molecules and stability and conformational order of the SAM. We have shown that the SAM can be formed by anodic oxidative adsorption, i.e., a reverse reaction of the reductive desorption, in an alkaline ethanolic solution containing thiol and the coverage of SAM increased and the peak position shifted negatively with adsorption time and the rates of formation and the conformational order of the SAM are higher in the decanethiol solution of higher concentration $[39,40]$.

In this work, we studied in detail the SAM formation process of decanethiol on $\mathrm{Au}(111)$ surface in ethanol solution containing decanethiol using electrochemical reductive desorption methods. The adsorption rate constant was determined and the effects of decanethiol concentration and temperature on the SAM formation process were investigated for a wide range of concentration $(0.1 \mu \mathrm{M} \sim 500 \mu \mathrm{M})$ and temperature 
$(253 \mathrm{~K} \sim 298 \mathrm{~K})$ of C10SH solution. A model for the thiol SAM formation is proposed.

\section{Experimental Section}

Reagent grade n-decanethiol $(\mathrm{C} 10 \mathrm{SH})$ and semiconductor grade $\mathrm{KOH}$ were purchased from Aldrich and reagent grade ethanol and $\mathrm{H}_{2} \mathrm{SO}_{4}$ were purchased from Wako Pure Chemicals. They were used without further purification. Water was purified using a Milli-Q water purification system (Yamamoto, WQ-500). Ultrapure Ar gas (99.9995\%) was purchased from Air Water.

A gold single crystal was prepared from a gold wire $(99.999 \%$ pure, $\phi=1 \mathrm{~mm}$, Tanaka Precious Metal) by the Clavilier method [42]. A Au(111) surface was obtained by cutting to parallel to the $\mathrm{Au}(111)$ facet using diamond saw (Buehler) and was then sequentially polished by $\# 400, \# 1000$ and $\# 2000$ polishing paper and $3 \mu \mathrm{m}$ and $0.5 \mu \mathrm{m}$ diamond slurry (Maruto Instrument). The $\mathrm{Au}(111)$ surface was flame annealed in a hydrogen flame and gradually cooled to room temperature in air prior to each measurement.

C10SH SAM was prepared by immersing the $\mathrm{Au}(111)$ substrate in ethanol solution containing various concentrations of $\mathrm{C} 10 \mathrm{SH}$ and at various temperatures for various period of time. The samples were rinsed by ethanol then by Milli-Q water immediately after that they were taken out from the thiol solution.

Temperature of thiol solution was controlled by Cryocool (NESLAB Instruments, Inc. CC-100 II).

Electrochemical measurements were carried out in a three-compartment electrochemical cell with a hanging meniscus configuration in a $0.5 \mathrm{M} \mathrm{KOH}$ aqueous solution using a potentiostat (Hokuto Denko, HA-151) and a function generator (Hokuto Denko, HB-111). Cyclic voltammograms (CVs) and linear sweep 
voltammograms (LSVs) were recorded using an X-Y recorder (Graphtec, WX1200). The electrode potential was referred to a $\mathrm{Ag} / \mathrm{AgCl}$ (saturated $\mathrm{NaCl}$ ) electrode, and a $\mathrm{Pt}$ wire was used as a counter electrode. The electrolyte solution was deaerated by bubbling Ar gas for at least $30 \mathrm{~min}$ before each experiment. The high quality and cleanliness of the $\mathrm{Au}(111)$ single crystal were ensured as $\mathrm{CV}$ s obtained in $0.1 \mathrm{M} \mathrm{H}_{2} \mathrm{SO}_{4}$ solution were in agreement with those for clean $\mathrm{Au}(111)$ electrodes reported previously $[43,44]$.

\section{Results and Discussion}

Concentration dependence Figure 1 shows typical LSVs of a Au (111) single crystal electrode, which was modified by being immersed in a $100 \mu \mathrm{M} \mathrm{C10SH}$ ethanol solution for various period of time at room temperature, obtained by sweeping the potential from $0 \mathrm{~V}$ to $-1.2 \mathrm{~V}$ at the sweep rate of $10 \mathrm{mV} \mathrm{s}^{-1}$ in a $0.5 \mathrm{M} \mathrm{KOH}$ aqueous solution. A sharp reductive peak was observed between $-0.9 \mathrm{~V}$ and $-1.0 \mathrm{~V}$. Peak area increased and peak potential shifted negatively with immersion time. This result is in good agreement with our previous result of the potentiostatic SAM formation in $\mathrm{C} 10 \mathrm{SH}$ ethanol solution, where it was found that area and position of reductive peak increased and shifted negatively, respectively, with the time kept at SAM formation potential, $+0.1 \mathrm{~V}$ [40].

Reductive charge was estimated by integrating current - potential relation. Figure 2 shows the reductive charge as a function of immersion time for SAMs prepared in $\mathrm{C} 10 \mathrm{SH}$ ethanol solution of various concentration at room temperature. Reductive charge increased with immersion time and reached to $75-80 \mu \mathrm{C} \mathrm{cm}^{-2}$ [45].

The charge-immersion time relations in relatively short immersion time were shown in Figure 2(b). It is clear that the reductive charge increased faster when the solution concentration was higher. Similar result was observed in the case of potentiostatic SAM 
where it was found that area of the reductive desorption peak increased faster with the time kept at SAM formation potential, $+0.1 \mathrm{~V}$, as the solution concentration was higher $[40]$.

Figure 3 shows the reductive desorption peak potential as a function of immersion time for the SAMs prepared in $\mathrm{C} 10 \mathrm{SH}$ ethanol solution of various concentrations at room temperature. Reductive desorption peak shifted negatively with immersion time and reached a constant value of ca. $-1.01 \mathrm{~V}$ as shown in Figure 3(a) except for the case of $0.1 \mu \mathrm{M}$, where the ultimate peak potential was slightly more positive than others, ca. $-0.98 \mathrm{~V}$. These results suggest that the ordering of SAM continued as SAM formation proceeded and highly ordered SAM was obtained after prolonged immersion regardless of solution concentration except for the SAM formation in $0.1 \mu \mathrm{M}$ solution. It is clear from Figure 3(b) that the peak potential shifted faster when the solution concentration was higher as expected. Similar trend was observed in the case of potentiostatic SAM formation [40].

Figure 4 shows the relation between reductive desorption peak potentials and the reductive charge obtained for all SAMs. There seems to be a universal relation between the peak potential and the reductive charge, showing that the peak potential, i.e., the quality of the SAM, is determined by adsorbed amount of the thiol, except for the SAMs formed in $0.1 \mu \mathrm{M}$ thiol solution. Reductive peak potential at a given coverage of the SAM formed in $0.1 \mu \mathrm{M}$ was relatively positive compared to those formed in thiol solutions of higher concentration. This may be due to larger amount of contamination and/or defects in the SAM because of the much longer formation time to reach the same coverage.

Kinetic analysis Many models have been proposed in previous reports as mentioned before. For example, some report said SAM formation is diffusion controlled [23, 
28-31] and the other said SAM formation followed Langmuir adsorption kinetics [13-15, $21,22,24,25,27,32,33]$.

Here we first assume the SAM formation process can be described by simple Langmuir adsorption kinetics in the initial stage as described by:

$$
\frac{d \theta}{d t}=k c(1-\theta)
$$

where $\theta$ is the normalized monolayer coverage, which is obtained from reductive desorption charge using the following equation, $c$ is the thiol concentration and $k$ is the rate constant.

$$
\theta=\frac{Q}{Q_{\max }}
$$

where $Q$ is the desorption charge at a given time and $Q_{\max }$ is the maximum reductive charge [46].

By integrating Eq (1), one obtains

$$
\ln (1-\theta)=-k c t
$$

Actually, linear relations were obtained between $\ln (1-\theta)$ and immersion time for all concentrations when immersion time was short as shown in Figure 5 for the SAM formed in (a) $3 \mu \mathrm{M}$ and (b) $200 \mu \mathrm{M}$ C10SH ethanol solution as examples. Similar results were obtained in previous studies $[32,33]$. The slope should give $-k c$ and $k c$ is defined as an apparent rate constant, $k_{o b s}$. Figure 6 shows the relation between concentration and the apparent rate constant both in logarithmic scale. Although a linear relation between $k_{o b s}(=k c)$ and $c$, i.e., a linear relation between $\log k_{o b s}$ and $\log c$ with slope equal 1 is expected from $\mathrm{Eq}(3), k_{\text {obs }}$ became constant when the concentration was higher than $100 \mu \mathrm{M}$. This shows simple Langmuir adsorption kinetics cannot describe the SAM growth mechanism in all concentrations. Similar tendency was reported by Karpovich and Blanchard as initial rate adsorption of 
1-octanethiol on gold surface from n-hexane became independent of the thiol concentration as it was higher than $300 \mu \mathrm{M}$ [13]. They suggested the presence of intermediate state. Dannenberger et al. also reported the existence of a linear relation between $\ln (1-\theta) / t$, which is equivalent to $k_{o b s}$ in the present study, and solution concentration for docosanethiol SAM formation on gold from hexane solution $(0.5 \mu \mathrm{M}$ $\sim 10 \mu \mathrm{M})[22]$.

We now assume the presence of intermediate state prior to chemisorption step into account. There are several reports about existence of physisorbed phase based on STM [16-18], AFM [20] and TPD [47, 48] studies. Xu et al. have proposed a two step adsorption model based on AFM study: first physisorption state with thiol molecules lying down and then chemisorption state on the gold surface [20]. Thus, we analyze the kinetic date by considering the two step thiol adsorption process: first physisorption step and then chemisorption step as:

$$
\begin{aligned}
& \mathrm{C} 10 \mathrm{SH}+\mathrm{Au} \stackrel{k_{1}}{\longrightarrow} \mathrm{C} 10 \mathrm{SH}---\mathrm{Au} \\
& \mathrm{C} 10 \mathrm{SH}---\mathrm{Au} \stackrel{k_{2}}{\longrightarrow} \mathrm{C} 10 \mathrm{~S}-\mathrm{Au}
\end{aligned}
$$

where $\mathrm{C} 10 \mathrm{SH}---\mathrm{Au}$ and $\mathrm{C} 10 \mathrm{~S}-\mathrm{Au}$ are the physisorbed and chemisorbed state, respectively, and $k_{1}$ and $k_{2}$ are the rate constants for physisorption and chemisorption step, respectively. By solving these two differential equations, the time dependence of the coverage can be described by the following equations.

$$
\begin{aligned}
& \frac{d \theta_{1}}{d t}=k_{1} c\left(1-\theta_{1}-\theta_{2}\right)-k_{2} \theta_{1} \\
& \frac{d \theta_{2}}{d t}=k_{2} \theta_{1}
\end{aligned}
$$

where $\theta_{1}$ and $\theta_{2}$ are the coverage of physisorbed and chemisorbed molecules, respectively. Time dependent coverage of each phase can be described by the following equations. 


$$
\begin{aligned}
& \theta_{1}(t)=\frac{k_{1} c}{k_{1} c-k_{2}}\left(e^{-k_{2} t}-e^{-k_{1} c t}\right) \\
& \theta_{2}(t)=\frac{k_{1} c}{k_{2}-k_{1} c} e^{-k_{2} t}-\frac{k_{2}}{k_{2}-k_{1} c} e^{-k_{1} c t}+1
\end{aligned}
$$

Since the reductive charge should correspond to the amount of chemisorbed species, only time dependence of $\theta_{2}$ is considered.

When the thiol concentration is very low, $k_{1} c$ is smaller than $k_{2}$, and Eq (9) becomes Eq (10).

$$
\ln \left(1-\theta_{2}\right)=-k_{1} c t
$$

This is essentially the same as Eq (4) and the slope of the plot between $\ln \left(1-\theta_{2}\right)$ and $t$ gives $k_{1} c$ and the physisorption process of thiol on gold is the rate limiting step.

When the thiol concentration is very high, $k_{1} c$ is much bigger than $k_{2}$ and Eq (9) becomes Eq (11).

$$
\ln \left(1-\theta_{2}\right)=-k_{2} t
$$

This shows also a first order kinetics with the rate constant of $k_{2}$, i.e., the concentration independent rate constant. The slope of the plot between $\ln \left(1-\theta_{2}\right)$ and $t$ gives $k_{2}$ and the conversion of physisorbed state to chemisorption becomes the rate limiting step.

Eqs (10) and (11) show that one will expect a linear relation between $\ln (1-\theta)$ and time in all concentrations, but the slope should be $k_{1} c$ when the concentration is low and $k_{2}$ when the concentration is high. Thus, apparent rate constant linearly increases with concentration when concentration is low and reached saturated value when concentration becomes high as experimentally observed (Figure 6).

Since $k_{o b s}$ is given by $k_{1} c$ in low concentration, $\log k_{o b s}$ should be linearly related with $\log C$ with the slope of 1 and the intercept at $\log C=0$ should give $\log k_{1}$ as observed in Figure 6. The value of $k_{1}$ is calculated to be $4.9 \times 10^{4} \mathrm{M}^{-1} \mathrm{~min}^{-1}$.

In high concentration, $k_{o b s}$ become constant and is expressed as $k_{2}$, which can be 
calculated from the value of constant part of Fig. 6 as $8.4 \times 10^{14}$ molecules $\mathrm{cm}^{-2} \mathrm{~min}^{-1}$. This value is very similar to the rate constant for SAM formation in $500 \mu \mathrm{M} \mathrm{FcC11SH}$, in which chemisorption limited process is expected from the present result, determined by QCM as $1.2 \times 10^{15}$ molecules $\mathrm{cm}^{-2} \mathrm{~min}^{-1}[12]$.

Yang et al. [25] also reported the two-step SAM formation process based on SFG measurements. They assumed that at early stage of the self-assembly process, the surface is covered with molecules in the disordered (liquid) phase and when the disordered phase density reaches a critical level, the adsorbates segregate into regions of liquid (I) and solid (II) phases in coexistence, such that the total surface coverage is the sum of the surface coverage of these two phases. The model is similar to that of $\mathrm{Xu}$ et al. (20) and that of present work but they considered that the disordered (liquid) phase is formed by chemisorption based on the result that non-resonant signal of SFG increased with time in this stage. One of the possible reasons for this discrepancy is that Yang et al. measured SFG intensity under the flow of thiol solution so that the diffusion limited physisorption process becomes much faster.

Temperature dependence Although we have reported the effect of SAM formation temperature on the sizes of domains and pits of the thiol SAM by STM [49], effect of temperature on the SAM formation rate has not been well studied [15].

Figure 7 shows typical LSVs of a $\mathrm{Au}(111)$ single crystal electrode, which was modified by immersing in a $200 \mu \mathrm{M} \mathrm{C10SH}$ ethanol solution for $1 \mathrm{~min}$ at various temperatures, obtained by sweeping the potential from $0 \mathrm{~V}$ to $-1.2 \mathrm{~V}$ at sweep rate of 10 $\mathrm{mV} \mathrm{s}^{-1}$. Peak area increased and peak potential shifted negatively at higher temperature. Figures 8 and 9 show reductive charge as function of immersion time for SAMs prepared in $200 \mu \mathrm{M}$ and $1 \mu \mathrm{M}$ thiol solution, respectively, at various temperature. It is clear that the reductive charge increased faster when the solution temperature was 
higher as expected.

The proposed model based on the concentration dependencies of the SAM formation rate is supported by the results of temperature dependencies of the SAM formation rate in thiol solutions of high and low concentration. The rate constants for the initial step of the SAMs formed in $1 \mu \mathrm{M}$ and $200 \mu \mathrm{M}$ at various temperatures were calculated from Eqs (10) and (11), respectively. Figure 10 shows linear relations between $\ln k_{o b s}$ and $1 / \mathrm{T}$ for $1 \mu \mathrm{M}$ and $200 \mu \mathrm{M}$ C10SH ethanol solutions. From the slope of these relations, the activation energies for the SAM formation in $1 \mu \mathrm{M}$ and $200 \mu \mathrm{M}$ C10SH ethanol solutions were determined to be $10.9 \mathrm{~kJ} / \mathrm{mol}$ and $29.9 \mathrm{~kJ} / \mathrm{mol}$, respectively. The activation energy for the SAM formation in $1 \mu \mathrm{M} \mathrm{C10SH}$ ethanol solution is rather small, suggesting the diffusion limited process. It means that there is no activation barrier for the SAM formation in $1 \mu \mathrm{M}$ C10SH ethanol solution except for transportation of the thiol molecule in ethanol solution. Thus, the rate limiting step in solution of low thiol concentration is diffusion controlled physisorption step. Camillone also suggested the diffusion-limited growth in the low-concentration regime but mentioned that formation of any physisorbed initial phase is very rapid based on the results of SHG [23]. It must be noted that the SHG measurements were carried out under solution flow so that diffusion limited physisorption process became much faster. The activation energy for the SAM formation in $200 \mu \mathrm{M}$ C10SH ethanol solution was comparable to the values of activation barrier for chemisorption of ethanethiol, butanethiol, hexanethiol, and decanethiol, which were determined by TPD to be about $29 \mathrm{~kJ} / \mathrm{mol}$ irrespective to the chain length [48]. Similar value was also reported by Dubois et al. [50].

\section{Conclusions}


The formation kinetics of decanethiol SAMs on $\mathrm{Au}(111)$ single crystal surfaces in ethanol solutions of very wide ranges of thiol concentration and temperature were investigated by electrochemical reductive desorption in $0.5 \mathrm{M} \mathrm{KOH}$ solution. Reductive charge of desorption peak increased with immersion time and reached the saturated value of $75-80 \mu \mathrm{C} \mathrm{cm}{ }^{-2}$ for all concentrations. Reductive peak potential shifted negatively with immersion time and reached $-1.01 \mathrm{~V}$. The adsorption rate of $\mathrm{C} 10 \mathrm{SH}$ on gold was faster in solutions of higher thiol concentration. Kinetic model is developed by assuming that SAM formation takes place by two steps, which consist of the physisorption step and the following chemisorption step. The rate limiting steps for the SAM formation in the C10SH solution of low and high $(>100 \mu \mathrm{M}) \mathrm{C} 10 \mathrm{SH}$ concentrations are the diffusion controlled physisorption and the chemisorption, respectively, and the activation energies are determined to be $10.8 \mathrm{~kJ} / \mathrm{mol}$ and 29.9 $\mathrm{kJ} / \mathrm{mol}$ for the former and the latter, respectively.

\section{Acknowledgment}

The present work was partially supported by Grants-in-Aid for Scientific Research from the Ministry of Education, Culture, Sports, Science and Technology (MEXT), Japan (13304047). YH is supported by Monbukagakusho (MEXT) scholarship. 


\section{References}

[1] J. Sagiv, J. Am. Chem. Soc. 102 (1980) 92.

[2] A. Ulman, Chem. Rev. 96 (1996) 1533.

[3] F. Schreiber, Progress in Surface Science 65 (2000) 151.

[4] R. G. Nuzzo, D. L. Allara, J. Am. Chem. Soc. 105 (1983) 4481.

[5] M. D. Porter, T. B. Bright, D. L. Allara, C. E. D. Chidsey, J. Am. Chem. Soc. 109 (1987) 3559.

[6] P. E. Laibinis, G. M. Whitesides, D. L. Allara, Y.-T. Tao, A. N. Parikh, R. G. Nuzzo, J. Am. Chem. Soc. 113 (1991) 7152.

[7] K. Uosaki, Y. Sato, H. Kita, Langmuir 7 (1991) 1510.

[8] K. Uosaki, Electrochemistry (Tokyo) 67 (1999) 1105.

[9] M. A. Bryant, J. E. Pemberton, J. Am. Chem. Soc. 113 (1991) 8284.

[10] Y. Sato, B. L. Frey, R. M. Corn, K. Uosaki, Bull. Chem. Soc. Jpn. 67 (1994) 21.

[11] F. Bensebaa, R. Voicu, L. Huron, T. H. Ellis, E. Kruus, Langmuir 13 (1997) 5335.

[12] K. Shimazu, I. Yagi, Y. Sato, K. Uosaki, Langmuir 8 (1992) 1385.

[13] D. S. Karpovich, G. J. Blanchard, Langmuir 10 (1994) 3315.

[14] W. Pan, C. J. Durning, N. J. Turro, Langmuir 12 (1996) 4469.

[15] H. M. Schessler, D. S. Karpovich, G. J. Blanchard, J. Am. Chem. Soc. 118 (1996) 9645.

[16] N. Camillone III, P. Eisenberger, T. Y. B. Leung, P. Schwartz, G. Scoles, G. E. Poirier, M. J. Tarlov, J. Chem. Phys. 101 (1994) 11031.

[17] R. Yamada, K. Uosaki, Langmuir 13 (1997) 5218.

[18] R. Yamada, K. Uosaki, Langmuir 14 (1998) 855.

[19] C. A. Alves, E. L. Smith, M. D. Porter, J. Am. Chem. Soc. 114 (1992) 1222.

[20] S. Xu, S. J. N. Cruchon-Dupeyrat, J. C. Garno, G.-Y. Liu, G. K. Jennings, T.-H. 
Yong, P. E. Laibinis, J. Chem. Phys. 108 (1998) 5002.

[21] M. Buck, F. Eisert, J. Fischer, M. Grunze, F. Trager, Appl. Phys. A 53 (1991) 552.

[22] O. Dannenberger, M. Buck, M. Grunze, J. Phys. Chem. B 103 (1999) 2202.

[23] N. Camillone Ш, Langmuir 20 (2004) 1199.

[24] M. Himmelhaus, F. Eisert, M. Buck, M. Grunze, J. Phys. Chem. B 104 (2000) 576.

[25] C. S.-C. Yang, L. J. Richter, J. C. Stephenson, K. A. Briggman, Langmuir 18 (2002) 7549.

[26] C. D. Bain, E. B. Troughton, Y.-T. Tao, J. Evd, G. M. Whitesides, R. G. Nuzzo, J. Am. Chem. Soc. 111 (1989) 321.

[27] A. Jakubowicz, H. Jia, R. M. Wallace, B. E. Gnade, Langmuir 21 (2005) 950.

[28] K. A. Peterlinz, R. Georgiadis, Langmuir 12 (1996) 4731.

[29] L. S. Jung, C. T. Campbell, J. Phys. Chem. B 104 (2000) 11168.

[30] F. S. Damos, R. C. S. Luz, L. T. Kubota, Langmuir 21 (2005) 602.

[31] R. Subramanian, V. Lakshminarayanan, Electrochim. Acta 45 (2000) 4501.

[32] G. A. Campbell, R. Mutharasan, Langmuir 21 (2005) 11568.

[33] H. G. Hong, W. C. Park, Electrochim. Acta 51 (2005) 579.

[34] T. Kakiuchi, H. Usui, D. Hobara, M. Yamamoto, Langmuir 18 (2002) 5231.

[35] C. A. Widrig, C. Chuang, M. D. Porter, J. Electroanal. Chem. 310 (1991) 335.

[36] D. F. Yang, C. P. Wilde, M. Morin, Langmuir 12 (1996) 6570.

[37] D. F. Yang, C. P. Wilde, M. Morin, Langmuir 13 (1997) 243.

[38] T. Kawaguchi, Y. Yasuda, K. Shimazu, M. D. Porter Langmuir 16 (2000) 9830.

[39] T. Sumi, H. Wano, K. Uosaki, J. Electroanal. Chem. 550-551 (2003) 321.

[40] T. Sumi, K. Uosaki, J. Phys. Chem. B 108 (2004) 6422.

[41] I. Thom, M. Buck, Surf. Sci. 581 (2005) 33.

[42] J. Clavilier, R. Faure, G. Guinet, R. Durand, J. Electroanal. Chem. 107 (1980) 205. 
[43] H. Angerstein-Kozlowska, B. E. Comway, A. Hamelin, L. Stoicoviciu, J.

Electroanal. Chem. 228 (1987) 429.

[44] A. Hamelin, J. Electroanal. Chem. 407 (1996) 1.

[45] This values were smaller than the values for the reductive desorption of a SAM with the saturated coverage of a $(\sqrt{3} \times \sqrt{3}) R 30^{\circ}$ structure $[18,35,36,38,39]$. This discrepancy may be caused by an error in the estimation of real surface area. We employed a hanging meniscus configuration both for surface area determination by oxide reduction and reductive desorption. Since the unmodified Au is hydrophilic but the SAM modified $\mathrm{Au}$ is hydrophobic, the real contact area of the electrode to the solution might have been smaller at the modified electrode, although we tried to make the contact area the same. This error might be substantial as the size of the electrode was very small.

[46] For the determination of $\theta$ using Q, one needs to use the charge corresponding to Faradaic desorption process but since various assumptions are involved in the estimation of capacitive component in the experimentally observed charge, it is very difficult to accurately determine the charge corresponding to the Faradaic process and, therefore, we used total charge obtained experimentally. Since both the capacitive and Faradaic components are related with the coverage, $\theta$ is reasonably described by Eq. (2) even if the total charge was used. Furthermore, effect of the error involved in $\theta$ on rate constant is relatively small as the slope of $\ln (1-\theta)$ vs. time plot is used to determine the rate constant.

[47] F. Schreiber, A. Eberhardt, T. Y. B. Leung, P. Schwartz, S. M. Wetterer, D. J. Lavrich, L. Berman, P. Fenter, P. Eisenberger, G. Scoles, Phys. Rev. B 57 (1998) 12476. [48] D. J. Lavrich, S. M. Wetterer, S. L. Bernasek, G. Scoles, J. Phys. Chem. B 102 (1998) 3456. 
[49] R. Yamada, H. Wano, K. Uosaki, Langmuir 16 (2000) 5523.

[50] L. H. Dubois, B. R. Zegarski, R. G. Nuzzo, J. Chem. Phys. 98 (1993) 678. 


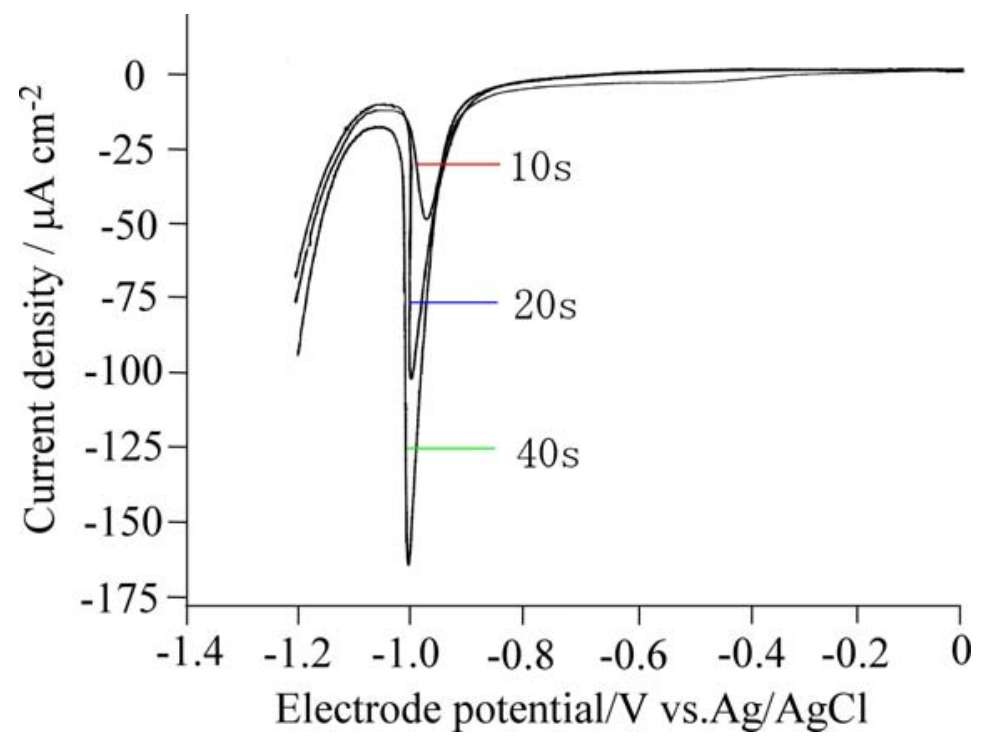

Figure 1. Current-voltage curves of $\mathrm{Au}$ (111) single crystal electrode, which was modified by immersing in ethanol solution containing $100 \mu \mathrm{M} \mathrm{C10SH}$ for various time periods at room temperature, measured in $0.5 \mathrm{M} \mathrm{KOH}$. Sweep rate : $10 \mathrm{mV} / \mathrm{s}$. 
(a)

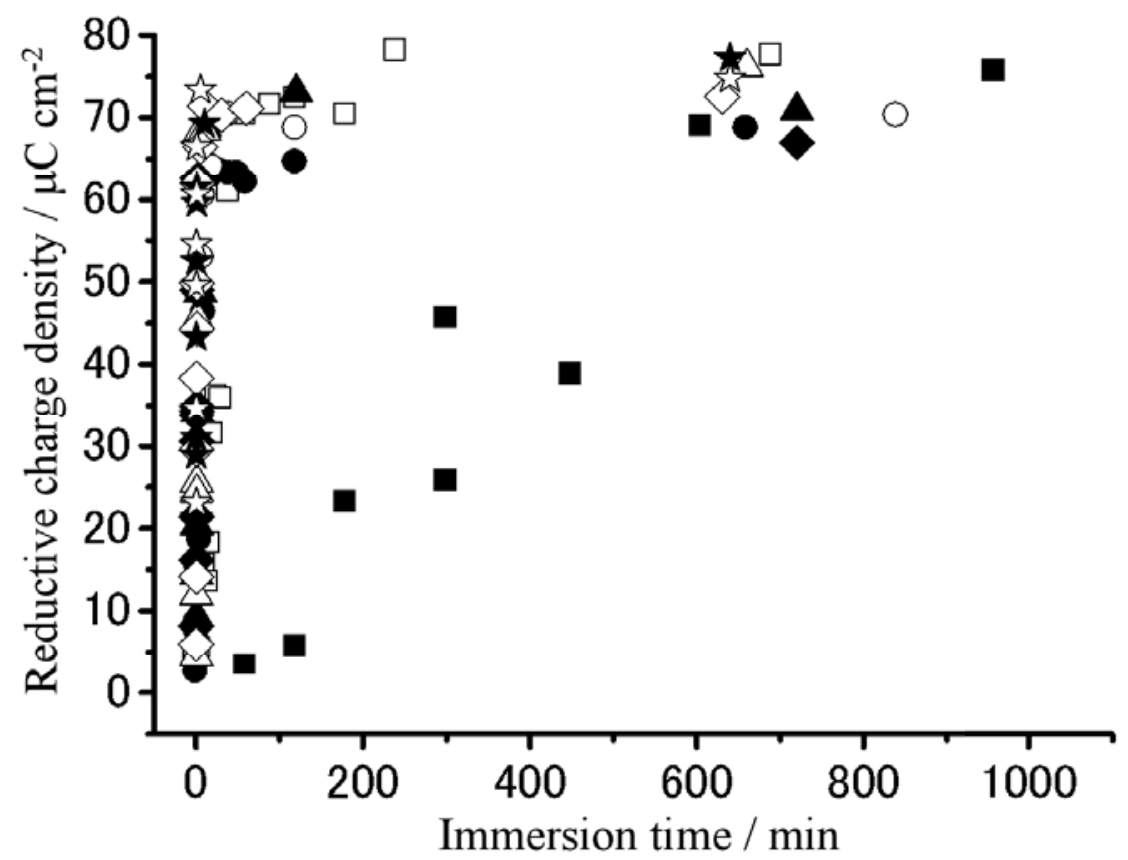

(b)

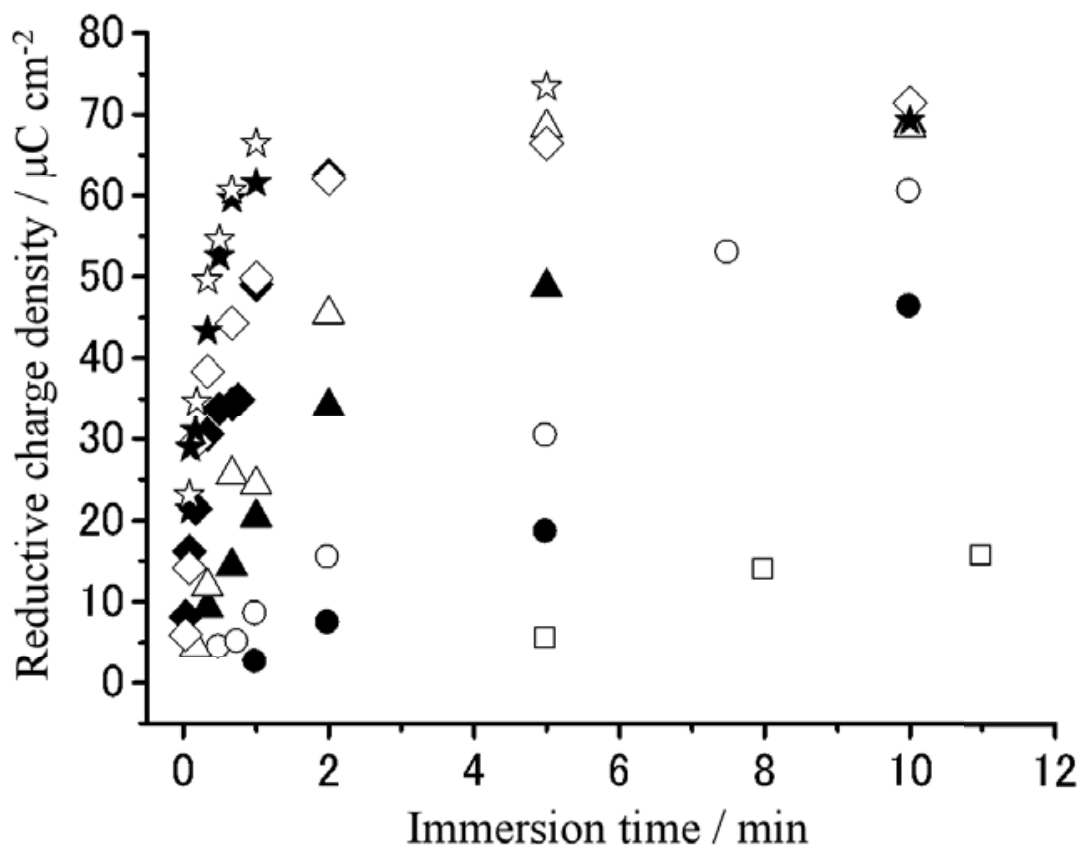

Figure 2. Reductive charge as a function of immersion time for SAMs prepared in 0.1 $\mu \mathrm{M}(\boldsymbol{\square}), 1 \mu \mathrm{M}(\square), 2 \mu \mathrm{M}(\boldsymbol{\bigcirc}), 3 \mu \mathrm{M}(\mathrm{O}), 5 \mu \mathrm{M}(\mathbf{\Delta}), 10 \mu \mathrm{M}(\Delta), 50 \mu \mathrm{M}(\diamond), 100$ $\mu \mathrm{M}(\diamond), 200 \mu \mathrm{M}(\star)$, and $500 \mu \mathrm{M}(\boldsymbol{\star}) \mathrm{C} 10 \mathrm{SH}$ ethanol solutions at room temperature. 
(a)

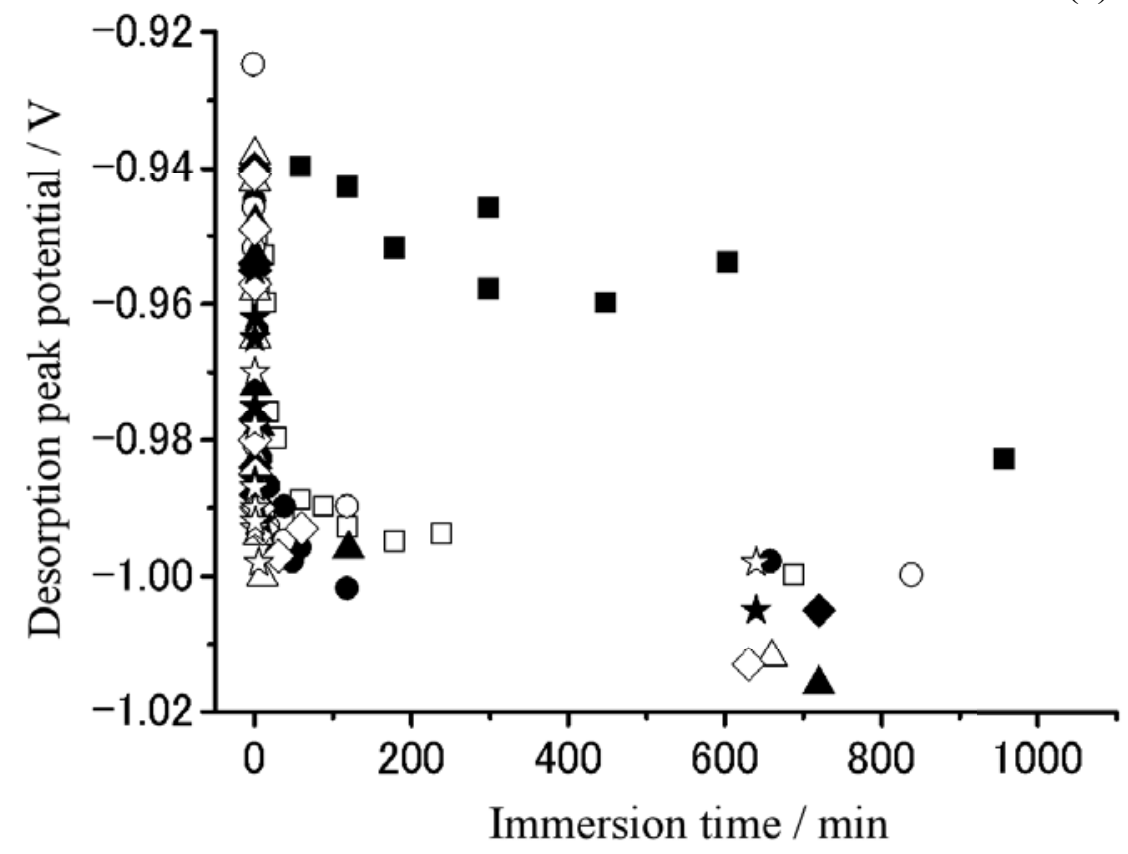

(b)

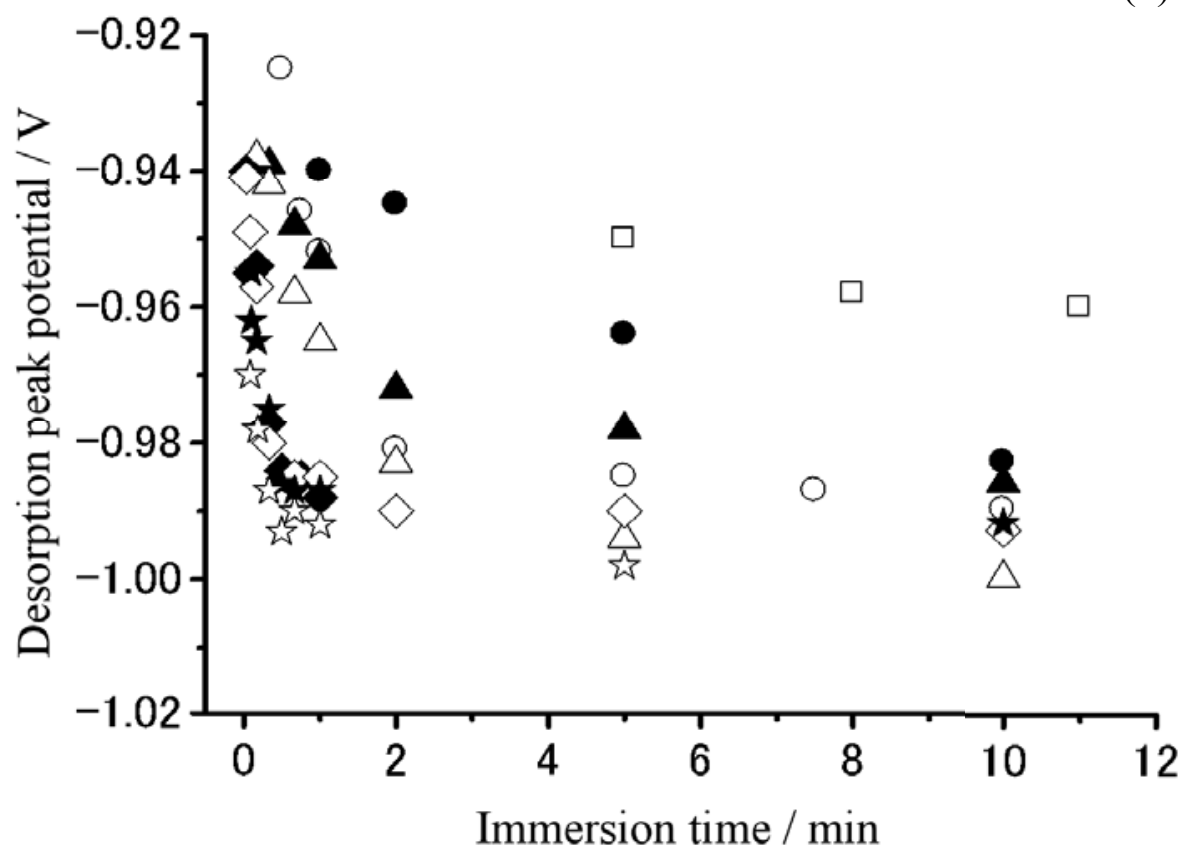

Figure 3. Reductive desorption peak potential as a function of immersion time for the SAMs prepared in $0.1 \mu \mathrm{M}(\boldsymbol{\square}), 1 \mu \mathrm{M}(\square), 2 \mu \mathrm{M}(\boldsymbol{O}), 3 \mu \mathrm{M}(\mathrm{O}), 5 \mu \mathrm{M}(\boldsymbol{\Delta}), 10 \mu \mathrm{M}$ $(\Delta), 50 \mu \mathrm{M}(\diamond), 100 \mu \mathrm{M}(\diamond), 200 \mu \mathrm{M}(\star)$ ), and $500 \mu \mathrm{M}(\boldsymbol{\varsigma}) \mathrm{C} 10 \mathrm{SH}$ ethanol solutions at room temperature. 


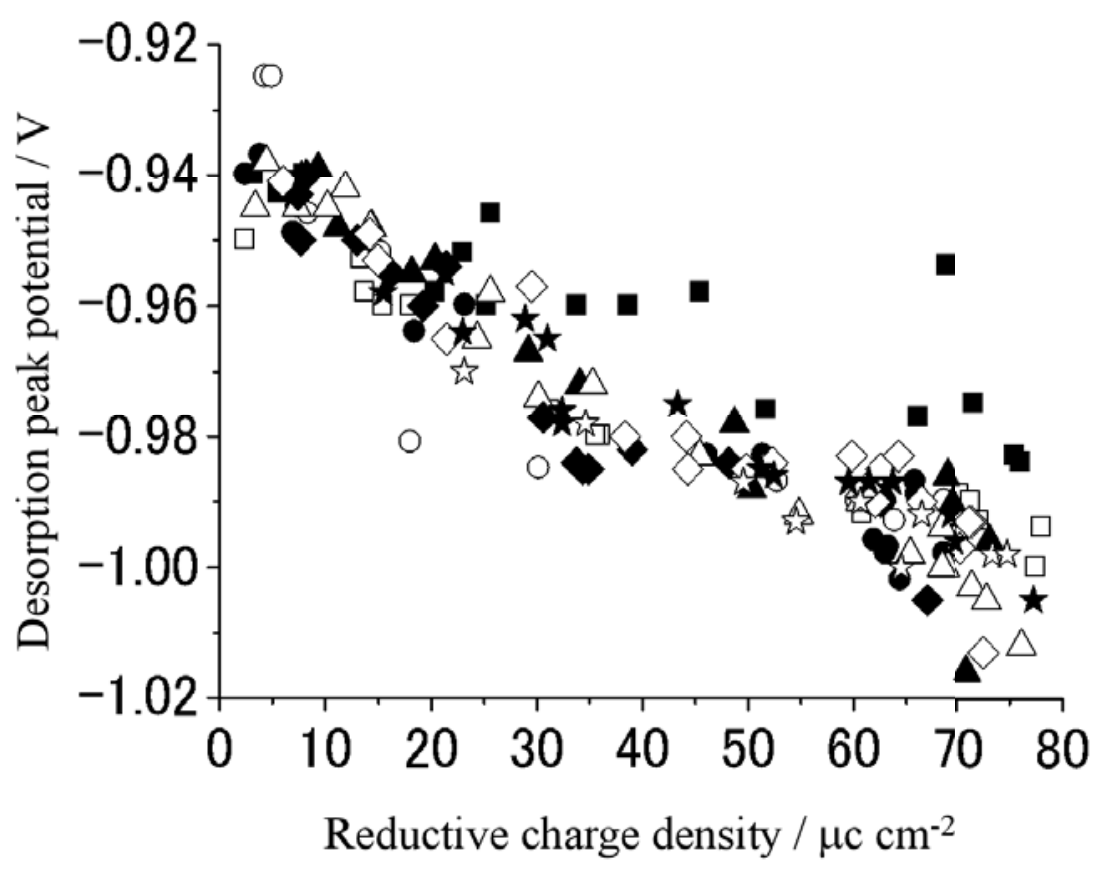

Figure 4. Relation between reductive desorption peak potentials and the reductive charge obtained for SAMs prepared in $0.1 \mu \mathrm{M}(\boldsymbol{\square}), 1 \mu \mathrm{M}(\square), 2 \mu \mathrm{M}(\mathbf{O}), 3 \mu \mathrm{M}$ $(\bigcirc), 5 \mu \mathrm{M}(\boldsymbol{\Delta}), 10 \mu \mathrm{M}(\Delta), 50 \mu \mathrm{M}(\diamond), 100 \mu \mathrm{M}(\diamond), 200 \mu \mathrm{M}(\star)$, and $500 \mu \mathrm{M}$ (支) $\mathrm{C} 10 \mathrm{SH}$ ethanol solutions at room temperature.
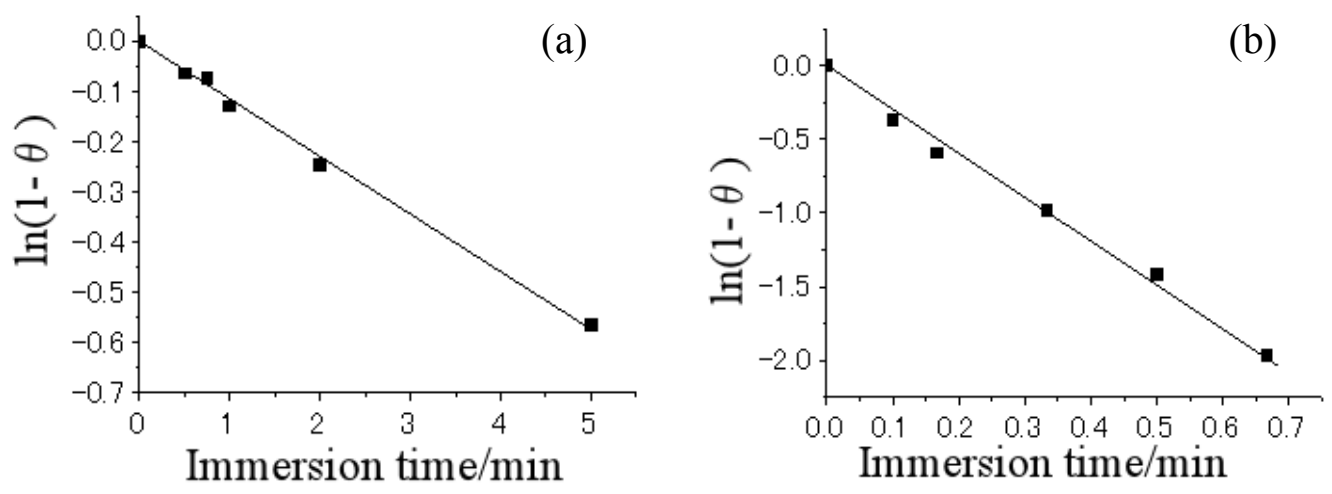

Figure 5. Immersion time dependencies of $\ln (1-\theta)$ of SAM prepared in (a) $3 \mu \mathrm{M}$ and (b) $200 \mu \mathrm{M} \mathrm{C10SH}$ ethanol solutions at room temperature. 


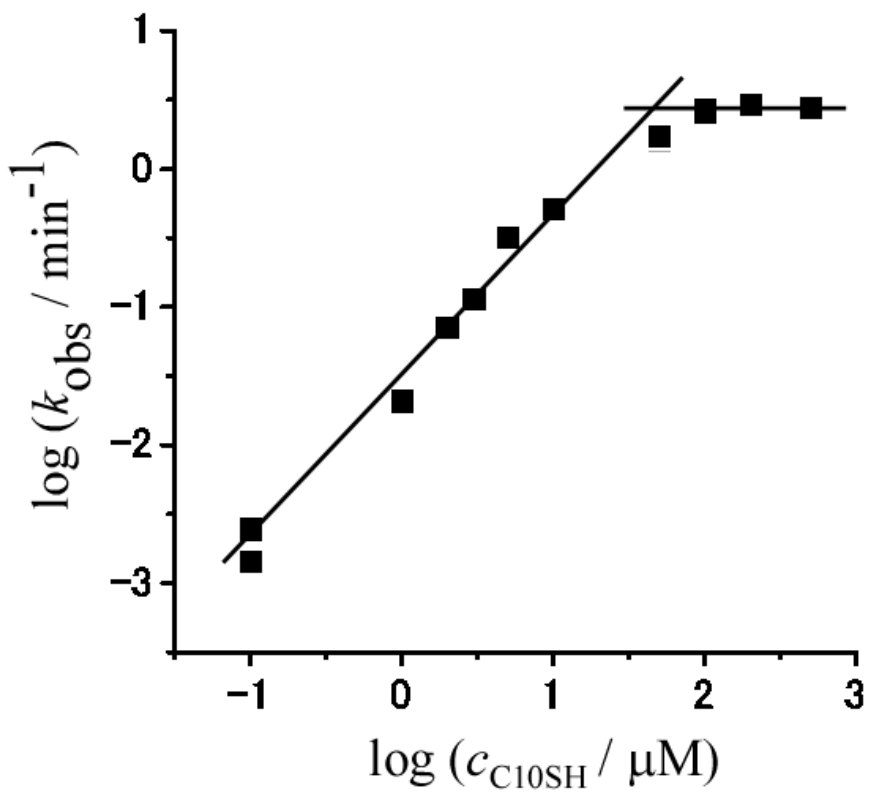

Figure 6. Relation between $\log \left(\mathrm{k}_{\mathrm{obs}}\right)$ and $\log ($ concentration of $\mathrm{C} 10 \mathrm{SH})$. 


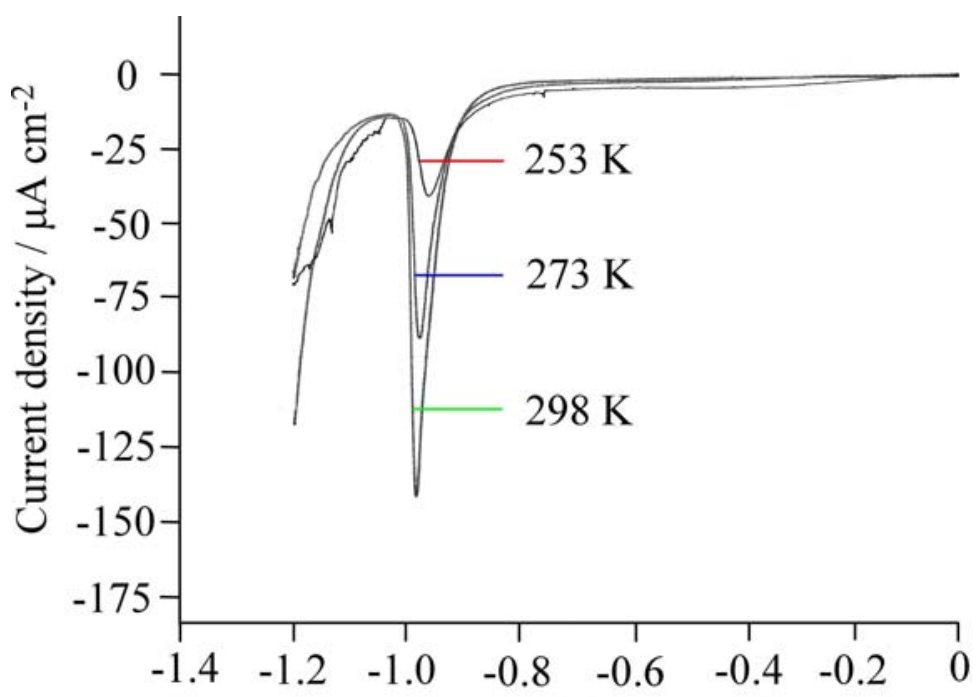

Electrode potential/V vs.Ag/AgCl

Figure 7. Current-voltage curves of $\mathrm{Au}$ (111) single crystal electrode, which was modified by immersing in ethanol solution containing $200 \mu \mathrm{M} \mathrm{C10SH}$ at various temperatures for $1 \mathrm{~min}$, measured in $0.5 \mathrm{M} \mathrm{KOH}$. Sweep rate : $10 \mathrm{mV} / \mathrm{s}$.

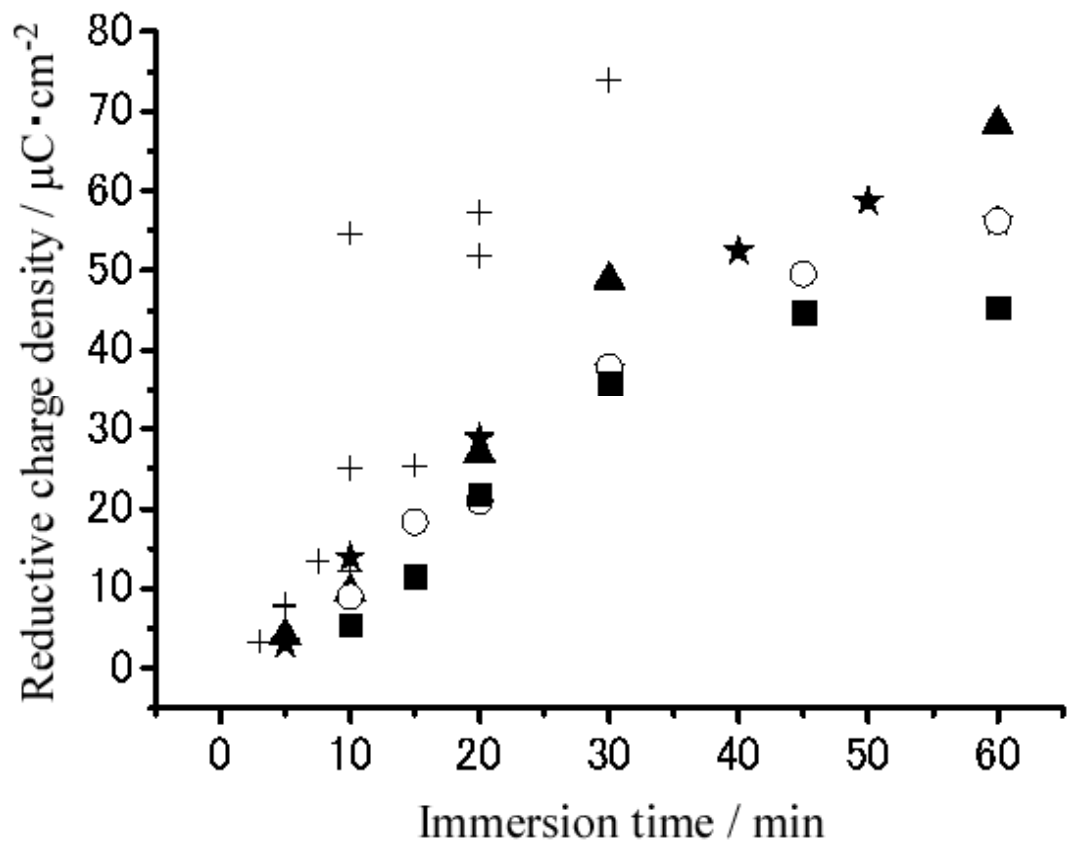

Figure 8. Reductive charge as a function of immersion time for SAMs prepared in 1 $\mu \mathrm{M}$ C10SH ethanol solution at $253 \mathrm{~K}(\boldsymbol{\square}), 261 \mathrm{~K}(\bigcirc), 273 \mathrm{~K}(\boldsymbol{\Delta}), 280 \mathrm{~K}(\star), 298 \mathrm{~K}$ $(+)$. 


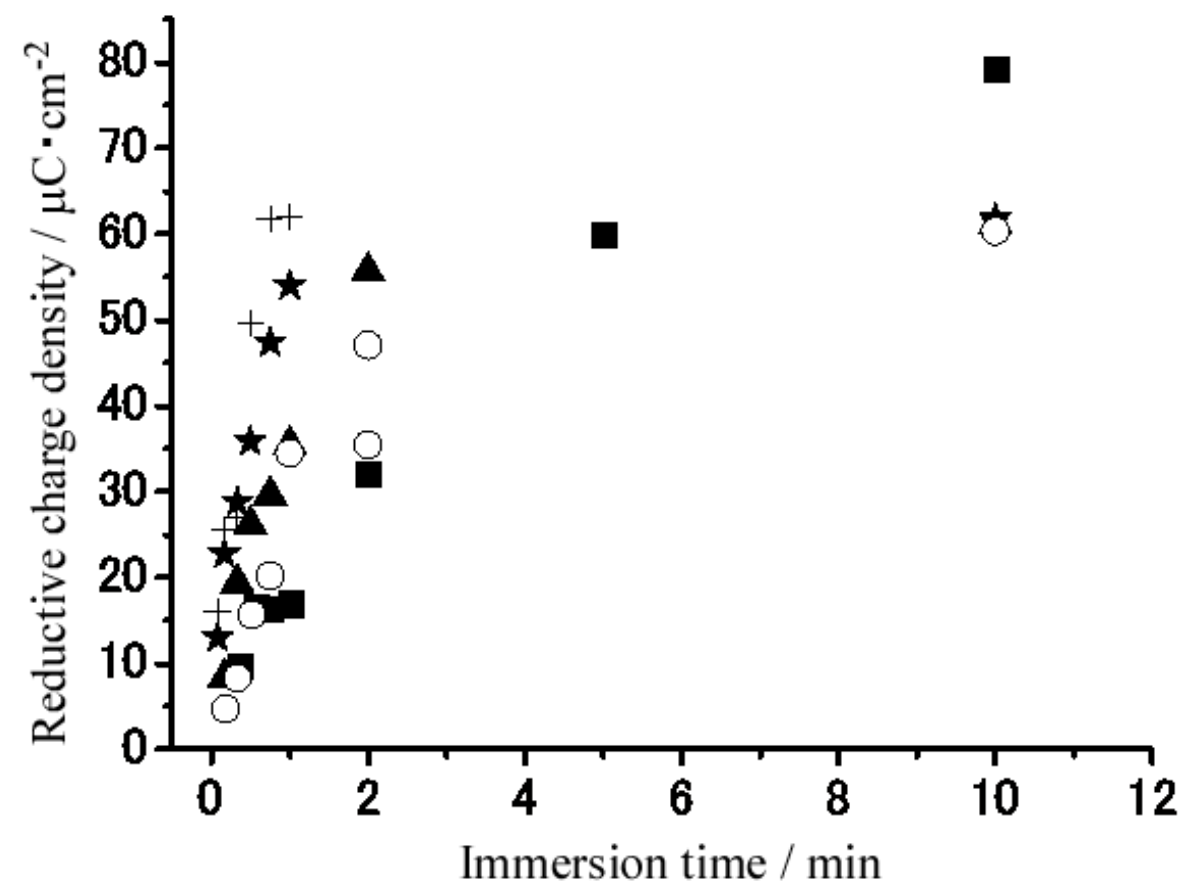

Figure 9. Reductive charge as a function of immersion time for SAMs prepared in 200 $\mu \mathrm{M}$ C10SH ethanol solution at $253 \mathrm{~K}(\boldsymbol{\square}), 261 \mathrm{~K}(\bigcirc), 273 \mathrm{~K}(\boldsymbol{\Delta}), 280 \mathrm{~K}(\star)), 298 \mathrm{~K}$ $(+)$.

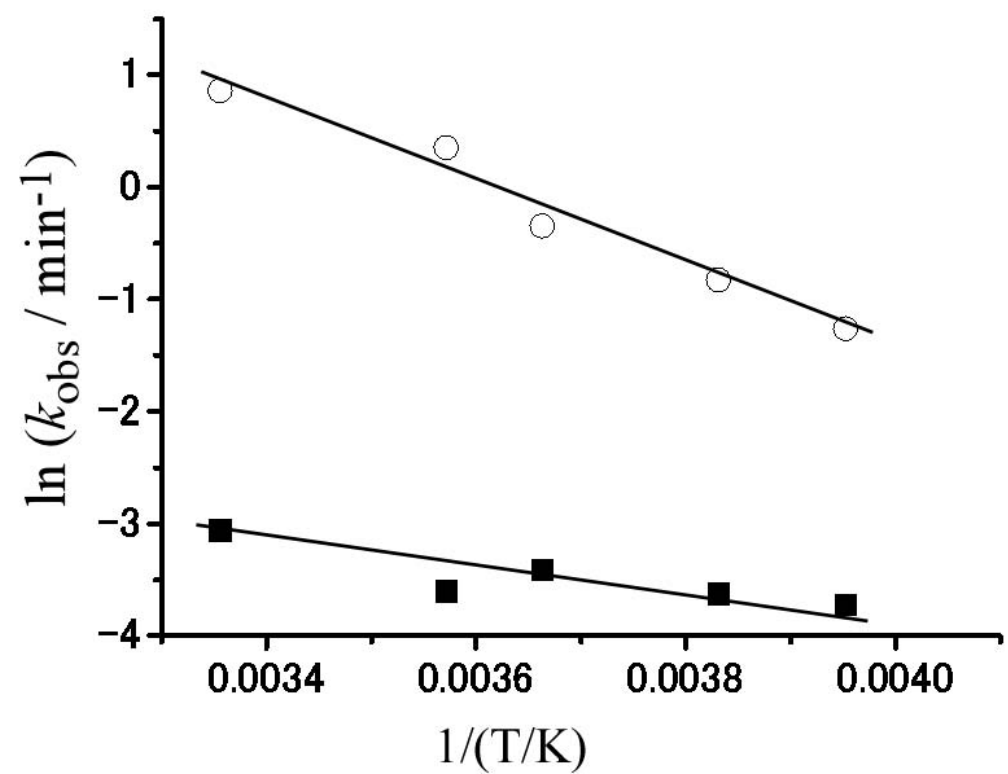

Figure 10. Temperature dependencies of the SAM formation rate constant in $1 \mu \mathrm{M}$ (ם) and $200 \mu \mathrm{M}(\bigcirc) \mathrm{C} 10 \mathrm{SH}$ ethanol solution. 